\title{
NON-TRAUMATIC DISPLACEMENT OF THE ATLANTO-AXIAL JOINT
}

\author{
A Report of Seven Cases
}

\author{
G. A. Hunter, Oxford, England
}

From the Orthopaedic Department of the University of the West Indies, Kingston, Jamaica

Displacement of the atlanto-axial joint was first described by Bell in 1830. Eriksson (1960) stated that over 200 cases have been reported.

The seven cases presented here show some interesting features and confirm the views of previous authors, who stated that the condition is much more common than was initially assumed. All our cases were seen within two years and were studied by me during a year spent in Jamaica.

The radiological discovery of displacement of the atlanto-axial joint should be interpreted in the light of clinical findings, and by itself should not lead to over-enthusiastic treatment, for the condition may be no more than a variant of normal.

Sullivan (1949) subdivided displacement at this joint into three types: 1) anterior unilateral: the head is rotated away from, but tilted towards, the affected side; 2) posterior unilateral: the head is rotated towards the affected side, and the anterior arch of the atlas is impalpable through the posterior pharyngeal wall; 3 ) anterior bilateral: the head is tilted forwards, and there is little or no rotation of the neck.

Berkheiser and Seidler (1931) pointed out that posterior bilateral displacement is theoretically impossible in the presence of an intact dens [odontoid process] and transverse ligament. By far the commonest clinical presentation is that of anterior unilateral displacement (Werne 1957), and the following cases all show this type.

\section{CASE REPORTS}

Case 1-A girl of ten complained of a stiff neck for three months. On examination, movements of the neck were limited in all directions. There was mild impairment of postural

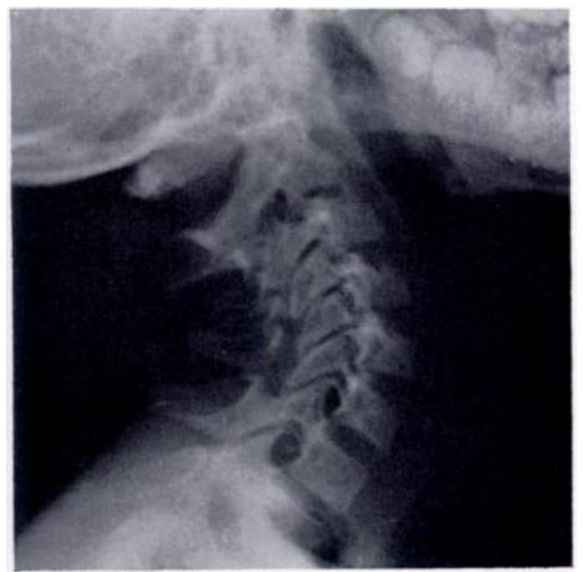

Fig. 1

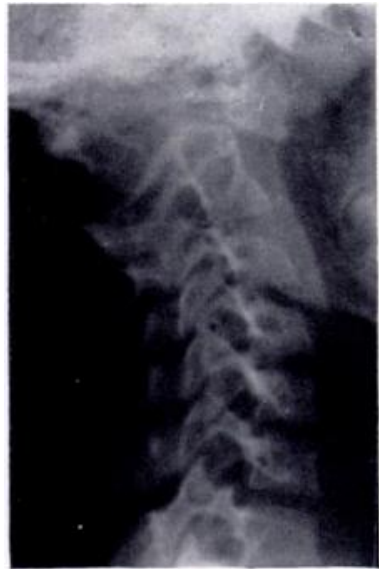

FIG. 2

Case 1. Radiographs to show the mobility of the atlas in flexion and extension. Figure 1-Extension. Figure 2-Flexion.

sense in the left upper limb. Radiographs showed anterior atlanto-axial displacement and tomographs confirmed the presence of the dens. Figures 1 and 2 show the range of movement of the atlas upon the axis during flexion and extension. 
Operation was advised because of the severe degree of displacement and the mild neurological signs. Posterior spinal fusion of the first and second vertebrae was performed using wire and autogenous bone grafts. Convalescence was uneventful. The neurological signs disappeared within six weeks, and reduction was demonstrated radiographically. The patient has remained without symptoms for six months.

Case 2-A boy of five complained of a stiff neck for three months. On examination, torticollis was present with limitation of all movements of the neck. Radiographs showed anterior atlanto-axial displacement. The patient was treated by cervical

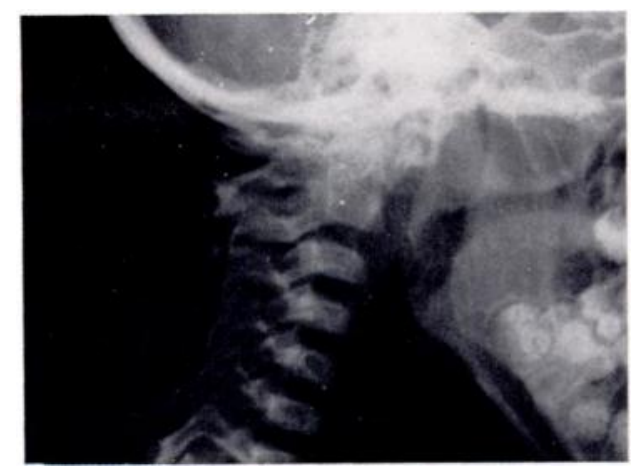

Fig. 3

Case 2-To show displacement of the atlantoaxial joint still present eighteen months after conservative treatment. traction for six days followed by a Minerva plaster for two weeks. The symptoms continued, and he was readmitted for further care, but discharged again because of the threat of a hurricane.

Eighteen months later he was found to have no symptoms, though there was slight limitation of rotation of his head to the left. Radiographs showed the displacement to be still present (Fig. 3).

Case 3-A girl of eighteen complained of neck pain for six months and weakness in the left side of the body for a week. On examination, all movements of the neck were limited. There were signs of an upper motor neurone lesion on the left side and impaired temperature sensation on the right side. She also had sickle-cell anaemia (haemoglobin 6-8 grammes

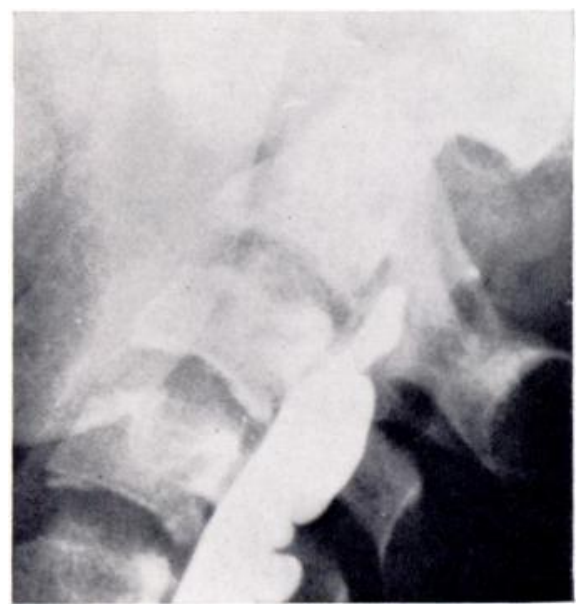

FIG. 4

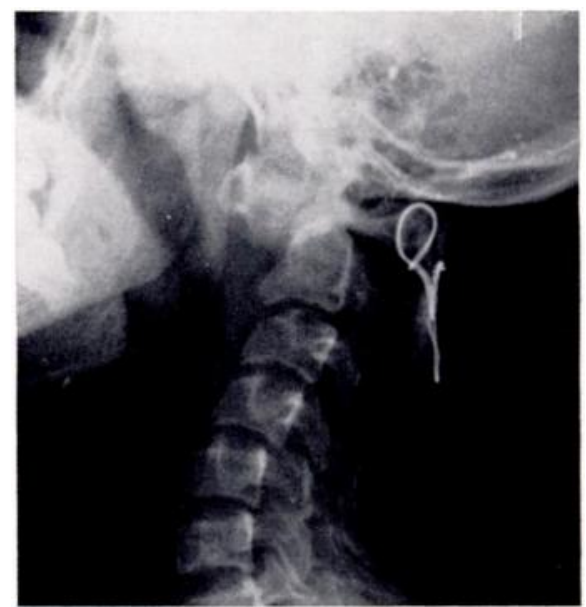

FIG. 5

Case 3. Figure 4-Myelograph showing obstruction at the level of the first and second cervical vertebrae. Figure 5-Displacement of atlanto-axial joint still present after spinal fusion.

per 100 millilitres; haemoglobin electrophoresis SS). Radiographs showed severe anterior displacement of the first and second cervical vertebrae with myelographic evidence of obstruction at the same level (Fig. 4).

She was treated by skull traction for two weeks, with rapid improvement of the neurological signs and symptoms. Posterior fusion of the first and second cervical vertebrae was performed using wire and autogenous bone grafts. Elective tracheostomy was performed at the end of the operation. 
Six weeks later the only abnormality was slight diminution of power in the left hand. This returned to normal after nine months, though the dislocation was not reduced (Fig. 5).

Case 4-A boy of eighteen months was paralysed for three days after a minor fall. He made a complete recovery at home.

When he was two, he was admitted with weakness of both arms and inability to walk for three weeks. On examination, there was generalised limb hypotonia; reflexes were absent and plantar responses were flexor. Anterior atlanto-axial displacement was diagnosed radiologically but, because his symptoms cleared up spontaneously in a few weeks, no further active measures were taken.

When he was eight, he had another similar attack. Examination revealed slight weakness of the left upper limb and both lower limbs with impaired sensation in the lower limbs. The right plantar response was extensor.

Radiographs showed unchanged atlanto-axial displacement as well as subluxation of the second cervical vertebra on the third. Tomographs showed posterior angulation of the

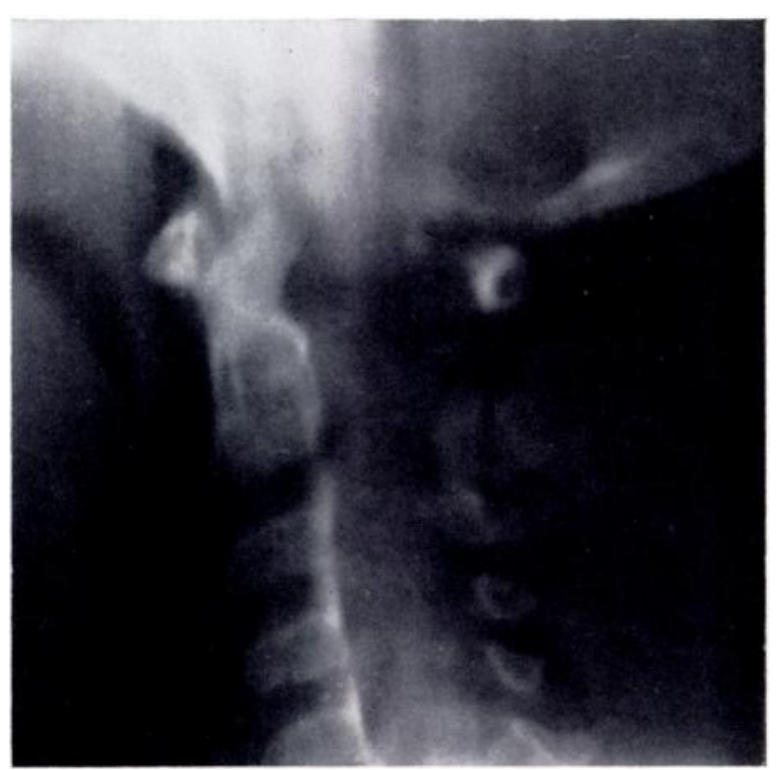

FIG. 6

Case 4-Tomograph to show backward angulation of the dens.

dens (Fig. 6). This could have represented an old fracture, or an epiphysial separation; Blockey and Purser (1956) stated that fractures of the dens in children up to the age of seven always represented epiphysial separation.

Because of a third episode of compression of the spinal cord, posterior spinal fusion of the first and second cervical vertebrae was performed. The patient was fit and well after six months; radiographs showed successful reduction of the first and second cervical vertebrae, but subluxation of the second on the third cervical vertebra persisted.

Case 5-A girl of seven developed torticollis two days after the extraction of a septic tooth. Two months after this episode a radiograph of her neck showed no abnormality (Fig. 7). Four months later a severe degree of anterior atlanto-axial displacement was present (Fig. 8). She 
was treated in a Minerva plaster for three months and after its removal the degree of displacement was judged to be less on radiographs (though the films are not entirely comparable).

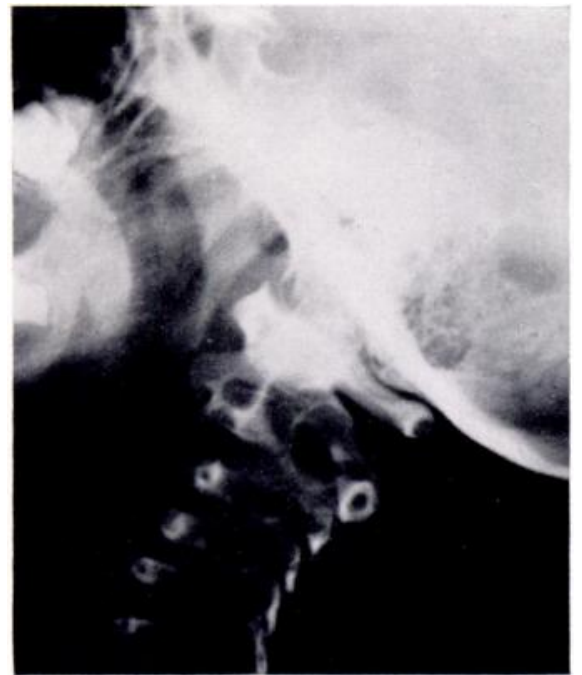

FIG. 7

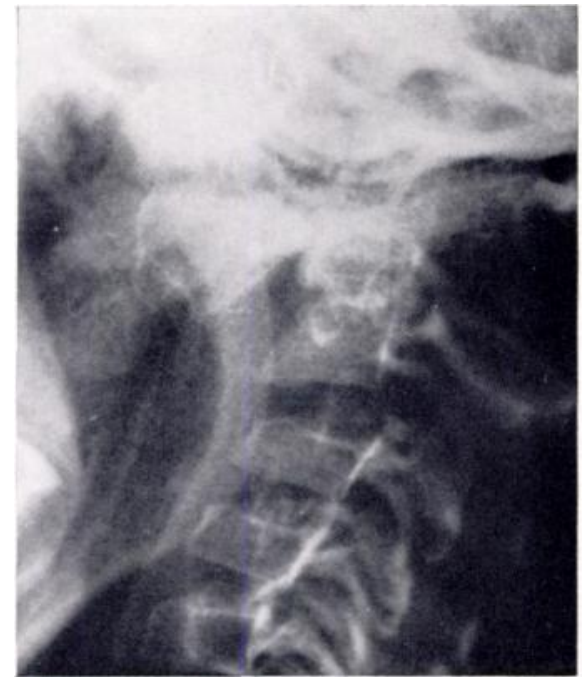

Fig. 8

Case 5. Figure 7-Showing no abnormality of the first and second cervical vertebrae. Figure 8 -Showing severe displacement of the atlas four months later.

This case is of interest in that, if it were not for the initial normal plain radiograph, it would have been said that the displacement had been caused by indirect trauma or adjacent sepsis. Immobilisation for three months produced symptomatic relief, but the displacement was still present.

Case 6-A boy of six complained of a stiff neck for twelve days. On examination, all movements of the neck were limited, but there was no neurological abnormality. Radiographs showed atlanto-axial displacement (Fig. 9). He was treated with skull traction for one week, and then a Minerva plaster for three weeks, and was discharged with a cervical collar. $\mathrm{He}$ did not attend again for three years. He had no symptoms and neck movements were full. Radiographs showed the displacement still present with anteroposterior movement of the anterior arch of the atlas, as seen in flexion-extension views (Figs. 10 and 11).

FIG. 9

Case 6-Showing severe displacement of the lateral mass of the atlas.

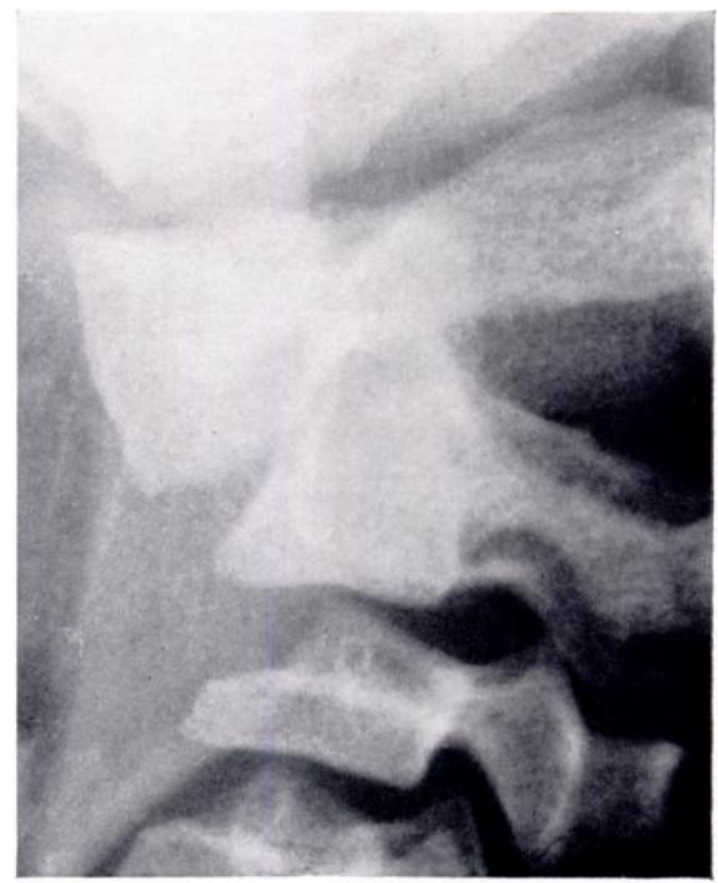

FIG. 9

VOL. $50 \mathrm{~B}$, NO. 1, FEBRUARY 1968 


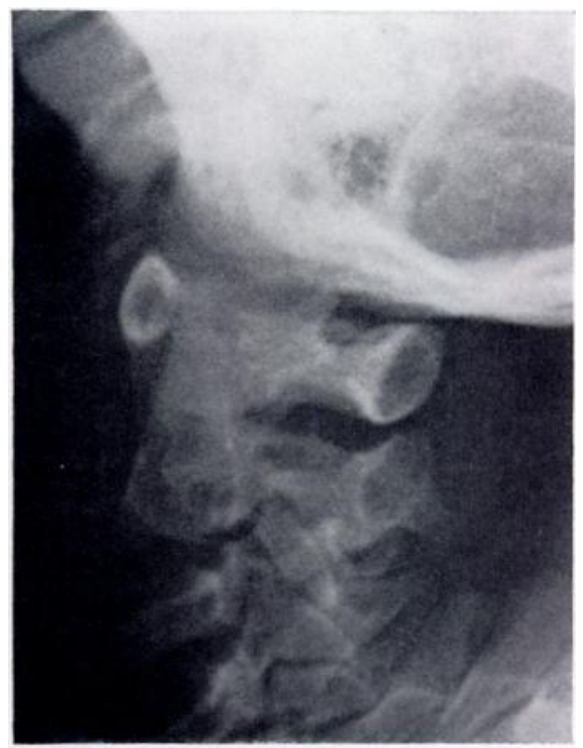

Fig. 10

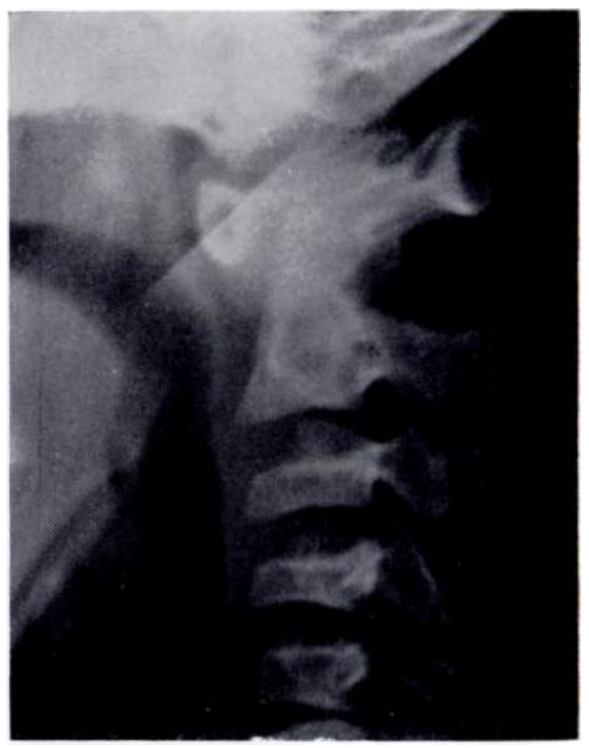

Fig. 11

Case 6. Figure 10-Showing the position of the atlas in extension. Figure 11-Showing the movement of the atlas on flexion.

Case 7-A boy of seven complained of a stiff neck for one month. On examination, there was torticollis to the left, and minimal upper motor neurone signs in the right upper limb. Radiographs showed atlanto-axial displacement with subluxation of the second on the third cervical vertebra. He was treated in a Minerva plaster for six weeks, and then a cervical collar for one month. Because we were becoming increasingly aware of this condition, it was decided to try to avoid operation. Six months later he was free of symptoms and there were no abnormal neurological signs. Radiographs still showed dislocation, with movement of the arch of the atlas in flexion-extension views.

\section{DISCUSSION}

Etiology-Wittek (1908) was the first to draw attention to the fact that displacement may follow local cervical infection. It is said that infection may predispose to displacement in various ways: 1) hyperaemic decalcification of bone; 2) softening of the transverse ligament and its attachments; 3 ) effusion into the atlanto-axial joints.

In favour of the infective hypothesis are the numerous case reports published in which there has been preceding cervical sepsis (Sullivan 1949). But there are arguments against it: 1) children of this age are constantly being troubled by cervical sepsis but atlanto-axial displacement is rare; 2 ) the time interval between the infection and the subsequent displacement seems to be too variable (for example one day (Watson Jones 1932) to several years (Boever and Hennebert 1953));3) there is no correlation with the severity of the infection.

There are several anatomical features of the atlanto-axial region in children which may predispose to displacement at the joint (Gray 1962). 1) The atlanto-axial joint has no inherent bony stability; 2) the capsule is loose in order to permit rotation; 3) there is great mobility at this level; 4) the facets of the upper cervical vertebrae are more horizontal than those lower down; the facets in younger children are more horizontal than those of older children (Sullivan, Bruwer and Harris 1958). This condition is most common in children between the ages of six and twelve (Watson-Jones 1955).

In relation to trauma, Werne (1957) found the cranio-vertebral ligaments to be of such strength that they could withstand a force greater than that capable of producing a cervical 
fracture. Coutts (1934) stated that chronic polyarthritis was a common cause of displacement of the atlas, but none of our reported cases showed any evidence of this disease.

Whatever the etiology of this condition, there must be some insufficiency of the transverse ligament to allow the atlas to slide forwards on the axis. Werne (1966) compared ropes with ligaments in support of his theory of acquired insufficiency of the transverse ligament. He said, "If you cut the cords of a rope on different levels, you can lengthen the rope (transverse ligament). If there are other ropes (alar ligaments) ready to take over the tension, you will never get a true rupture. Displacement is only possible if the transverse ligament has been divided or is completely ruptured, and if the alar and capsular ligaments allow the displacement to occur."

Various authors (Jackson 1950; Hohl 1964; Cattell and Filtzer 1965) have examined lateral radiographs of the atlanto-axial region in children during flexion and extension. Most of these authors agree that the distance between the anterior arch of the atlas and the dens in flexion is 2-2.5 millimetres in adults and 3 millimetres in children. Jackson, however, reported a child with a measured distance of 4.5 millimetres who was without symptoms.

Cattell and Filtzer (1965) examined a group of children between the ages of one and seven. They found that there was a distance of 3 millimetres or more in 20 per cent of a survey of seventy children. Six of these fourteen children also had overriding of the anterior arch of the atlas on top of the dens in extension, suggesting excessive mobility of the cervical spine in these children.

Sullivan et al. (1958) showed that hypermobility of the second or the third cervical vertebra is often seen in children (fifteen of 100 children showed marked subluxation of the second on the third cervical vertebrae).

All the cases studied in this paper showed marked mobility of the atlas in flexionextension views, and two of the seven showed subluxation of the second on the third cervical vertebrae. Displacement of the atlanto-axial joint in children may be a variant of normal in the early stages, and may be associated with hypermobility lower down the cervical spine. Diagnosis-The patient is usually a child between six and twelve (Watson-Jones 1955) although the condition has been reported in early infancy (Lounavaara 1949) and at the age of sixty-two (Wilson, Michele and Jacobson 1940). The sex incidence is approximately equal (Wilson et al. 1940).

The commonest presenting symptom is stiffness of the neck. There may be a history of preceding cervical sepsis (Coutts 1934). In anterior unilateral displacement the head may be turned away from the affected side. Movements of the neck are limited. Rarely there may be evidence of neurological compression (Sullivan 1949). If the displacement is anterior, the anterior arch of the atlas may be palpable through the posterior pharyngeal wall (Coutts 1934). Radiographs confirm the diagnosis. Coutts (1934), in a full account of the radiological features, stressed that the only diagnostic film was a lateral view of the neck in full flexion. An antero-posterior view may show spurious lateral displacement due to rotation of the dens (Werne 1957).

Differential diagnosis includes congenital or spasmodic torticollis, osteomyelitis of the cervical spine, trauma, cervical sepsis and adenitis, and congenital anomalies of the dens. If neurological features predominate, the possibility of syringomyelia, cerebellar tumour, bulbar palsy and myasthenia gravis should be considered.

Treatment. Reduction-Most of the authors referred to agree that skull traction with the head extended is the best method of treatment. The period for symptomatic improvement and radiological confirmation of reduction is usually between two and four weeks.

Fixation-Maintenance of reduction for between one and six months is advised. The use of a Thomas's collar or Minerva plaster is recommended.

Cervical fusion-There is little information about the advisability of this operation. Watson-Jones (1955) stated that one should not be forced into an extensive operation to treat

VOL. $50 \mathrm{~B}$, NO. 1, FEBRUARY 1968

D 
a benign condition. Eriksson (1960) stated that only five cases are on record in which the patients were operated on because of displacement without fracture or demonstrable anomaly. If operation is performed the advantage of limiting fusion to the first and second cervical vertebrae is that an almost normal range of movement is preserved.

Our own cases are unusual in that four of the seven showed evidence of neurological compression of varying severity. In Boever and Hennebert's (1953) series of 103 cases, only fifteen showed evidence of neurological compression; even in such cases the prognosis is good (Werne 1957), and patients with tetraplegia have been known to recover (Berkheiser and Seidler 1931; Watson Jones 1932; Wilson et al. 1940).

In Case 1 we were worried by the degree of displacement and neurological compression. Case 4 was remarkable in that there was complete spontaneous recovery after two episodes of tetraparesis. All patients are now symptomless; the radiographs show satisfactory operative reduction but perhaps, in retrospect, we operated unnecessarily. It would have been interesting to have seen the effect of more prolonged conservative treatment.

In Case 3 spinal fusion was considered essential after preliminary traction. The outcome has been relief of symptoms, but the radiographs still show considerable displacement. Similarly in Cases 2, 5, 6 and 7 the patients are now without symptoms after conservative treatment, varying from two weeks to three months, but the radiographs show that considerable displacement is still present.

If a diagnosis is made and the patient receives proper treatment, the outlook is favourable (Watson Jones 1932; Sullivan 1949). We would agree with the principles of reduction and fixation. Cervical fusion of the first and second vertebrae should be reserved for those patients who have severe atlanto-axial displacement and who show evidence of neurological compression which does not improve with conservative treatment.

\section{SUMMARY}

1. Seven cases of non-traumatic anterior atlanto-axial displacement in young people are recorded.

2. Four of the seven patients showed evidence of neurological compression. All cases improved symptomatically with treatment, but five still show persistent radiological displacement.

3. The mechanism of the displacement is thought to be due to acquired insufficiency of the transverse ligament.

4. The distance between the anterior arch of the atlas and the dens in children measured on lateral radiographs of the cervical spine, varies from two to more than three millimetres.

5. Skull traction followed by immobilisation in a collar or Minerva plaster is advised.

6. The place of cervical fusion is discussed.

My thanks are due to Professor J. S. R. Golding, under whose care these patients were treated, and to the photographic department of the Medical Faculty of the University of the West Indies.

\section{REFERENCES}

Bell, C. (1836): The Nervous System of the Human Body as explained in a series of papers read before the Royal Society of London. Case number 148, p. 403. London: Longmans.

Berkheiser, E. J., and Seidler, F. (1931): Nontraumatic Dislocations of the Atlanto-Axial Joint. Journal of the American Medical Association, 96, 517.

Blockey, N. J., and PURSER, D. W. (1956): Fractures of the Odontoid Process of the Axis. Journal of Bone and Joint Surgery, 38-B, 794.

Boever, F., and Hennebert, P. (1953): Les dislocations non traumatiques de la colonne cervicale. Revue de chirurgie orthopédique et réparatrice de l'appareil moteur, 39, 24.

Catrell, H. S., and Filtzer, D. L. (1965): Pseudosubluxation and Other Normal Variations in the Cervical Spine in Chidren. Journal of Bone and Joint Surgery, 47-A, 1295.

Coutrs, M. B. (1934): Atlanto-Epistropheal Subluxations. Archives of Surgery, 29, 297. 
ERIKsson, B. (1960): Operated Case of Recurrent Dislocation of the Atlas. Acta Orthopaedica Scandinavica, $29,85$. Gray, H. (1962): Gray's Anatomy. Thirty-third edition, p. 491. Edited by D. V. Davies and F. Davies. London: Longmans.

Hонь, M. (1964): Normal Motions in the Upper Portion of the Cervical Spine. Journal of Bone and Joint Surgery, 46-A, 1777.

JaCkson, H. (1950): The Diagnosis of Minimal Atlanto-Axial Subluxation. British Journal of Radiology, 23, 672.

JACOBS, C. N. (1918): Atlas and Axis Luxation. American Journal of Orthopaedic Surgery, 16, 357.

Jacobson, G., and AdleR, D. C. (1953): An Evaluation of Lateral Atlanto-Axial Displacement in Injuries of the Cervical Spine. Radiology, 61, 355.

LounavaARA, K. I. (1949): Forward Subluxation of the Atlas Following Birth Trauma. Acta paediatrica, 37, 341.

Sullivan, A. W. (1949): Subluxation of the Atlanto-Axial Joint: Sequel to Inflammatory Processes of the Neck. Journal of Pediatrics, 35, 451.

Sullivan, C. R., Bruwer, A. J., and Harris, L. E. (1958): Hypermobility of the Cervical Spine in Children: A Pitfall in the Diagnosis of Cervical Dislocation. American Journal of Surgery, 95, 636.

Watson-Jones, R. (1932): Spontaneous Hyperaemic Dislocation of the Atlas. Proceedings of the Royal Society of Medicine, 25, 586.

WATSON-JONES, R. (1955): Fractures and Joint Injuries. Fourth edition, volume II, p. 981. Edinburgh and London: E. \& S. Livingstone Ltd.

WERne, S. (1956): Spontaneous Atlas Dislocation. Acta orthopaedica Scandinavica, 25, 32.

Werne, S. (1957): Studies in Spontaneous Atlas Dislocation. Part 1. The Craniovertebral Joints. Acta orthopaedica Scandinavica, Supplement 23, 9.

Werne, S. (1966): Personal communication.

Wilson, M. J., Michele, A. A., and Jacobson, E. W. (1940): Spontaneous Dislocation of the Atlanto-Axial Articulation. Journal of Bone and Joint Surgery, 22, 698.

WitTEK, A. (1908): Ein Fall von Distensionsluxation im Atlanto-epistropheal-gelenke. Münchener Medizinische Wochenschrift, 55, 1836.

VOL. 50 B, NO. 1, FEBRUARY 1968 